\title{
Tracheal cancer - treatment results, prognostic factors and incidence of other neoplasms
}

\author{
Aleksandra Napieralska, Leszek Miszczyk, Stawomir Blamek \\ Department of Radiotherapy, Maria Skłodowska-Curie Memorial Cancer Center and Institute of Oncology, Gliwice Branch, \\ Poland
}

Radiol Oncol 2016; 50(4): 409-417.

Received 29 January 2016

Accepted 16 June 2016

Correspondence to: Stawomir Blamek, M.D., Department of Radiotherapy, Maria Skłodowska-Curie Memorial Cancer Center

and Institute of Oncology, Gliwice Branch, ul. Wybrzeże AK 15, 44-101 Gliwice, Poland. Phone: +48 322788 052; Fax: +48 322788001 ;

E-mail: blamek@gmail.com

Disclosure: No potential conflicts of interest were disclosed.

Background. Tracheal cancers (TC) are rare and treatment results that are reported are typically not satisfactory. The purpose of this research was assessment of the results of treatment of TC patients, identification of potential additional surgery candidates, evaluation of prognostic factors, and assessment of the occurrence of other malignancies. Patients and methods. The Regional Cancer Database and the Hospital Database were searched for patients with tracheal neoplasms. Fifty-eight of 418 patients identified initially, met the inclusion criteria (primary TC with confirmed histology and complete treatment records). Standard statistical tests were used.

Results. Squamous cell carcinoma (SCC; 63.8\%) and adenoid cystic carcinoma (ACC; 15.5\%) were the most commonly diagnosed histological types of TC. Radiotherapy was delivered in 48 cases, surgery or endoscopic resection in 20, and chemotherapy in 14. TC was diagnosed as a second cancer in 10 patients, in 1 patient it occurred prior to the lung cancer, and in 1 was diagnosed simultaneously. During the median follow-up of 12.7 months, $85.5 \%$ of the patients died because of the disease. Local recurrence occurred in $17 \%$ cases. In univariate analysis, patients with ACC had statistically better five-year overall survival (77.8\%) than those diagnosed with SCC $(8.4 \%, p=0.0001)$. Radiotherapy, performance status and haemoptysis were factors significantly influencing overall survival (OS) in the multivariate analysis. Among patients who were not treated surgically, 15-26\% were found to constitute additional surgery candidates, depending on the selection criteria.

Conclusions. The diagnostic workup should be focused on the identification of TC patients suitable for invasive treatment and radiotherapy. Respiratory system cancer survivors can be considered a risk group for tracheal cancer. Radiotherapy constitutes an important part of the treatment of patients with TC.

Key words: tracheal cancer; metachronous neoplasms; adenoid cystic carcinoma; squamous cell carcinoma; radiotherapy

\section{Introduction}

Primary tracheal carcinomas (TC) are rare and account for $0.01-0.4 \%$ of all malignancies with annual incidence of merely 0.2 cases per 100,000 people. ${ }^{1-4}$ In most patients, TC are symptomatic, but often misdiagnosed as asthma, chronic pulmonary disorder or tumours of the thyroid or lungs. ${ }^{3-12}$ Two most common histological types are squamous cell carcinoma (SCC), and adenoid cystic carcinoma
(ACC) - these two types account for over $75 \%$ of primary TC. $6,7,13,14$

Knowledge on tracheal cancer is limited only to the results of retrospective reviews of institutional and national databases. Most of the studies suggest that standard methods of treatment should include extensive segmental resection with adjuvant radiotherapy (RT) or chemoradiotherapy. ${ }^{1,3,4-7,13,15}$ Modern techniques of tracheal surgery are associated with low perioperative risk when performed 

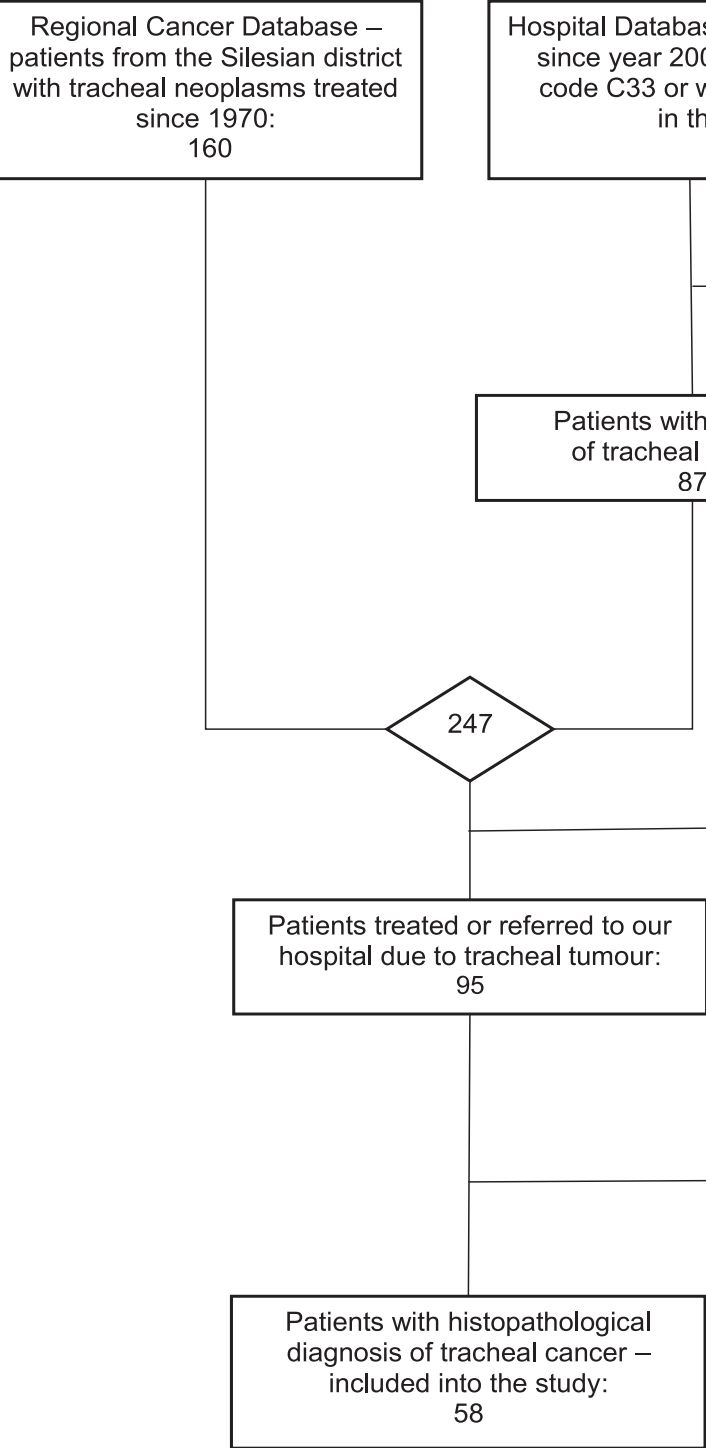

FIGURE 1. Patient identification algorithm and inclusion criteria

in specialized centres, and some researchers indicate that resection should be possible in more than $25-50 \%$ of patients. $3,14,16,17$

Adjuvant RT (3-6 weeks after the surgery with total dose of $60 \mathrm{~Gy}$ or a biologically equivalent dose of neutrons) or primary curative RT treatment in inoperable cases (doses ranging between 68 and 80 Gy) have also been proven effective in some studies. ${ }^{3,18,19}$ There is a lack of information on the late effects occurring after definitive RT for TC. The quantitative analysis of normal tissue effects in the clinic (QUANTEC) report suggests that limiting the dose to 80 Gy or lower using standard fractionation may reduce the risk of central airway stenosis. Therefore, most patients with TC are treated with lower doses (although some radioresistant tumours, such as ACC, may require higher doses to achieve local control and a small increase of the risk of treatment toxicity is acceptable). ${ }^{20}$

According to Grillo et al., approximately $40 \%$ of all tracheal SCCs are reported to occur before, together with or after carcinoma of the oropharynx, larynx, or lung. ${ }^{13}$ In spite of the above, second cancers are not very often described among patients with TC and only one large study (270 patients) reported second cancers in $19.6 \%$ of TC patients. $8,13,15,21-23$

The aim of our study was to assess the outcome of patients treated for TC, search for potential prognostic factors, evaluate the occurrence of second malignancies, and, finally, identify those pa- 
tients who potentially could have been additional surgery candidates.

\section{Patients and methods}

We conducted a retrospective study on patients diagnosed with TC in our institution between 1997 and 2015. The Regional Cancer Database and the Hospital Database were searched for patients with tracheal neoplasms. Among 418 patients (160 from the Regional Cancer Database and 258 from the Hospital Database), we found 58 cases that met the inclusion criteria (Figure 1).

Medical records of patients were reviewed to search for second malignancies. All patients with lung or larynx cancer invading trachea were excluded from the study. Second cancer was diagnosed if one of the following criteria were met: histopathology of the second cancer was different from tracheal cancer histopathology or disease-free interval between those two cancers exceeded three years.

Additional research was performed to identify those patients, who potentially could have been candidates for surgery. Our criteria for a theoretical surgery candidate were: [1] good performance status (Eastern Cooperative Oncology Group [ECOG] $0-1)$, [2] no nodal or distant metastases, [3] no infiltration of surrounding organs and [4] age below 70 (due to possible surgery contraindications not associated with TC).

The overall survival (OS) was evaluated using the Kaplan-Meier method. To verify the significance of variables influencing the OS in the univariate analysis, the Cox proportional hazard model was employed. $P$ of less than 0.05 value was considered to indicate statistical significance. Follow-up was defined as the period of time from the date of diagnosis of tracheal cancer to the date of the cut-off or death. The cut-off date for survival was December 14, 2015. Follow-up was calculated separately for the whole group and for patients who were alive at December 14, 2015. Time to progression was measured from the date of the end of the treatment to the date of local or distant progression. The study was performed according to the Helsinki Declaration and the Institutional Review Board Committee.

\section{Results}

\section{Patients' characteristics}

We found 58 cases diagnosed in 41 males and 17 females (gender ratio: 2.4:1). The age at diagnosis
TABLE 1. Distribution of histology types and methods of treatment summary

\begin{tabular}{lc}
\hline Histology & $\begin{array}{c}\text { Number of } \\
\text { patients }\end{array}$ \\
\hline Squamous cell carcinoma & $37(63.8 \%)$ \\
Adenoid cystic carcinoma & $9(15.5 \%)$ \\
Microcellular carcinoma & $5(8.6 \%)$ \\
Non-microcellular carcinoma & $4(6.9 \%)$ \\
Adenocarcinoma & $2(3.5 \%)$ \\
Papillary carcinoma & $1(1.7 \%)$ \\
\hline Methods of treatment & Number of \\
patients
\end{tabular}

varied from 27 to 79 , mean 57.6 ( $S D \pm 12.3$ ). The details of histopathological diagnoses and treatment employed are shown in Table 1.

Pathologic grade was poorly documented (19 cases) and in most cases for which it was reported the neoplasm was poorly differentiated or undifferentiated $(14 ; 74 \%)$. All patients were diagnosed on the basis of histopathological examination, bronchoscopy, and chest computed tomography (CT). In 50 cases, bronchoscopy or CT showed a mass protruding into tracheal lumen. Stricture of the trachea was found in 43 patients, whereas infiltration of surrounding tissues was present in 28 cases.

Fourteen patients had ECOG performance status of 0 , thirty three patients had the status of 1 , while in eleven cases it was 2 . Tumour size was difficult to assess due to retrospective nature of the study. The available data were obtained from CT scans and bronchoscopy. Only ten patients had a tumour smaller than $2 \mathrm{~cm}$ in its maximum diameter (T1 according to T-stage by Bhattacharyya). ${ }^{7}$ Nodal status was assessed in 57 of 58 (98.3\%) patients. In 23 of them, the lymph nodes were involved. Metastases in other organs were found in four patients before the treatment and occurred in eleven more patients during the follow-up. 
TABLE 2. The characteristics of radiotherapy

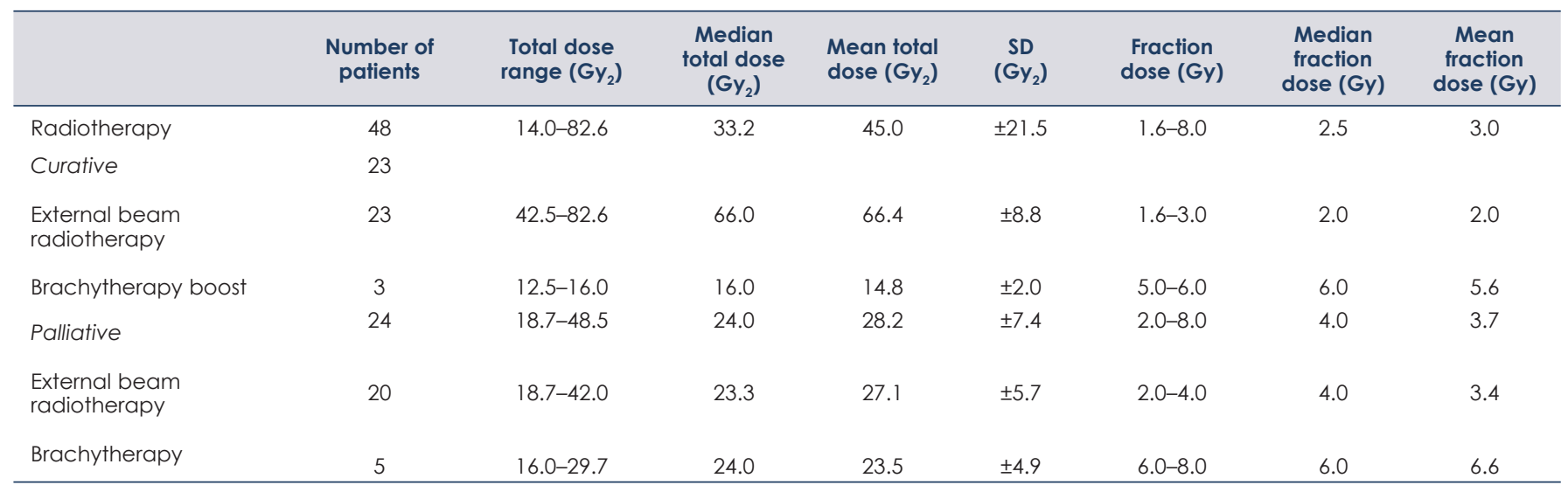

$\mathrm{Gy}_{2}=$ doses converted to 2-Grey equivalent doses (EQD2) using linear-quadratic model

\section{Symptoms}

Clinical symptoms occurred in $95 \%$ of patients. The most common symptom was dyspnoea $(72.4 \%)$, cough $(53.4 \%)$, haemoptysis (32.8\%), and hoarseness $(27.6 \%)$. Other symptoms included weight loss (22.4\%), pain (15.5\%), dysphagia (12.1\%), weakness $(10.3 \%)$, stridor $(5.2 \%)$, fever $(8.6 \%)$, the feeling of having an obstacle in trachea $(10.4 \%)$, and neck tumour $(6.9 \%)$. Forty-two patients $(72.4 \%)$ were current or former smokers, and among them 32 persons were diagnosed with SCC, 3 patients with ACC, 2 others with microcellular carcinoma, and 5 patients with other histological types. As for the number of pack-years, it varied from 8 to 135 with the median of 40 (mean 46.2).

\section{Treatment characteristics Radiotherapy}

Forty-eight out of 58 patients were treated with the ionizing radiation in our Institution. In 25 cases, RT was the only treatment modality used, 11 patients were irradiated with curative intent and 14 patients had palliative radiotherapy. Brachytherapy (BT) was a part of the treatment in 8 cases, and in 3 of them it was used as an additional boost to radical external beam RT. External beam radiotherapy was a part of therapy in 48 patients (Table 2).

To sum up the doses of various RT courses delivered with different fraction doses (FD), the physical doses were converted to 2-Gray equivalent doses (EQD2) using linear-quadratic model..$^{29} \mathrm{TD}$ $\left(\mathrm{Gy}_{2}\right)(\alpha / \beta=10)$ in palliative RT ranged from 18.7 to $48.5 \mathrm{~Gy}_{2}$ (mean 28.2; median 24) delivered in 2 to 16 fractions of 2.0 to $8.0 \mathrm{~Gy}$ (mean 4.1; median 4 ). In radical $\mathrm{RT}$, the fraction dose (FD) varied from 1.6 to 3 Gy (median 2) and the tumour was irradiated to TD ranging from 42.5 to $82.6 \mathrm{~Gy}_{2}$ (mean 66.4, median 66.0). TD of BT boost ranged between 10 and 12 Gy (EQD2 = 12.5-13.3) delivered in two fractions (FD 5-6 Gy). In all patients irradiated with curative or palliative intent, the treated volume included the tumour of trachea and margins added to create the clinical and planning target volumes. In 15 cases, lymph nodes were included in the clinical target volume (mediastinal lymph nodes in 9 cases; mediastinal and supraclavicular lymph nodes in 6 cases), and the treatment comprised of two phases - in the first phase the irradiated volume encompassed the primary tumour and lymph nodes with margins, whereas, in the second phase - only the tumour with margins. In one case, RT was delivered after radical surgery, and neck nodes were irradiated with TD of $42.5 \mathrm{~Gy}_{2}$. In one case, the exact data on the RT volume was not available.

Most of the patients treated with radiotherapy in our hospital had their treatment delivered with linear accelerators with 6-20 MV photons. Five patients treated in earlier years of the study (before 2004) were treated on Cobalt-60 machines. Six patients were irradiated outside our Institution and the detailed data on the treatment machines used were not available. Data on energies used in the treatment were available in 54 cases: fifteen patients received treatment with $6 \mathrm{MV}$ photons, sixteen persons with $20 \mathrm{MV}$ photons, three patients with 6 and $20 \mathrm{MV}$ photons, two patients with $15 \mathrm{MV}$ photons, and five others with 60-Cobalt (1.25 MeV). During the treatment, the position of the patient was verified based on skin marks prepared during simulation and MV portal imaging. Image guided radio- 
therapy (IGRT; orthogonal X-ray images acquired before every fraction) was used in later years of the study (after the year of 2006). Most of the patients had conventional, 3D conformal plans, four were treated with intensity modulated radiotherapy (IMRT), and one with tomotherapy.

Eight patients received high dose rate (HDR) brachytherapy with Iridium-192. All patients had bronchoscopy before application. The applicator was placed in close proximity of the tumour. The size of the applicator depended on tumour size and pattern of infiltration. In all the cases, the treated volume consisted of the gross tumour volume with a margin.

Radiation Therapy Oncology Group (RTOG) and the European Organization for Research and Treatment of Cancer (EORTC) criteria were used to assess toxicity of radiotherapy delivered. The entries in the medical records of the patients were re-evaluated, because in the majority of cases the radiation-induced reaction was rather presented in a descriptive form than given exactly according to the EORTC/RTOG grading system. Thirty-one patients did not declare any side effects. Eight reported dysphagia, three indicated dyspnoea, two patients suffered from oedema, additional two patients experienced pain and one person suffered from deterioration of the general condition. In most cases, the toxicity was minor (9 cases) or mild (6 patients), however one patient died due to haemorrhage in the course of brachytherapy.

\section{Surgery and systemic therapy}

Surgery or endoscopic resection of the tumour was combined with RT in 17 cases; however, only two patients had radical excision of the tumour. Thirteen patients had only endoscopic resection of the tumour. Seventeen patients had tracheotomy and stents placed inside the trachea as a part of the palliative treatment. Chemotherapy $(\mathrm{CTH})$ was applied in 14 cases (in 13 cisplatin-based), and in 5 of them it was the only treatment given. CTH regimens used included cisplatin alone or combined with: adriamycin, navelbine, etoposide, mitomycin $\mathrm{C}$ or 5 -fluorouracil. Patients received one to six cycles of CTH. As all the patients received CTH outside our institution, the assessment of toxicity of the systemic treatment could not have been performed.

\section{Follow-up}

The median follow-up of all patients was 12.7 months. The cut-off date for survival was December

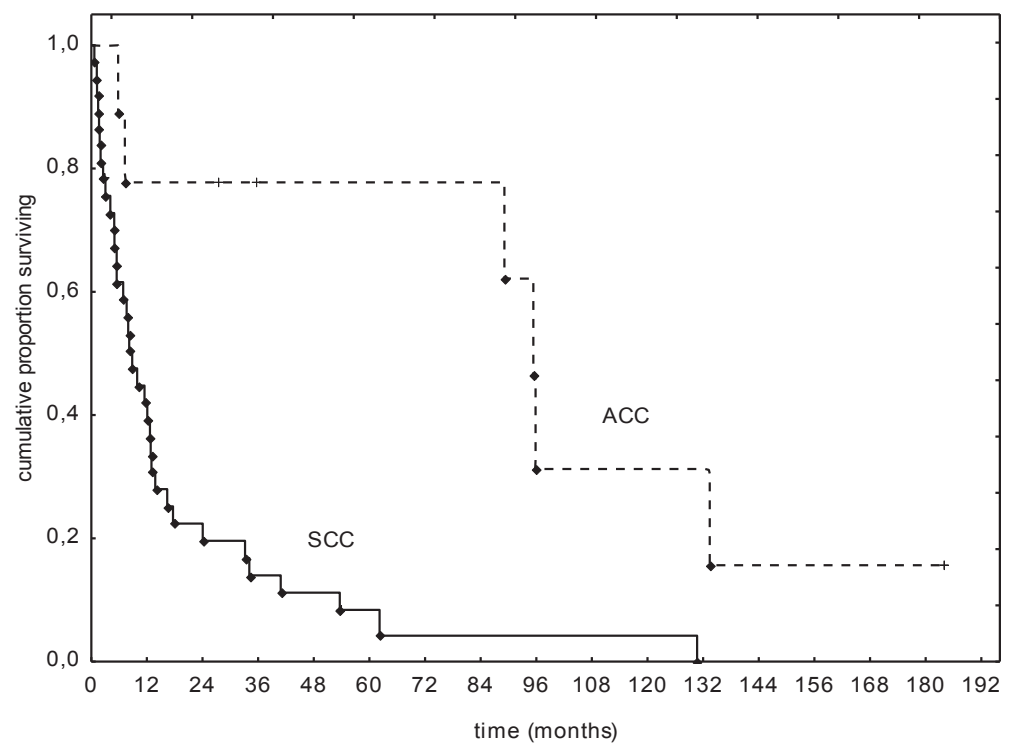

FIGURE 2. Overall survival of patients with adenoid cystic carcinoma (ACC) and squamous cell carcinoma (SCC).

14,2015 . Of 58 patients, 49 patients $(84.5 \%)$ died because of the disease and 9 were still alive. For the nine patients who were alive, the median followup was 27.4 months (range 0.5 to 183.8 months).

Thirty-two patients had control visits in our institution. Within this group, 11 cases of local recurrence of the disease from 4 to 67 months (median 49) after the treatment was observed. In 5 patients, it was a recurrence of SCC, in one patient - ACC, in one case microcellular carcinoma, in 2 cases adenocarcinoma, and in one patient - non-microcellular carcinoma. In all the cases of the recurrent disease, RT was a part of the therapy, whereas surgery was performed in 2 cases. BT was delivered in 3 cases as a part of the treatment of the recurrence. Five patients had been treated with cisplatin-based CTH, and three had symptomatic treatment.

A second recurrence was diagnosed in 5 cases. In 2 patients, BT was delivered, two had external beam radiotherapy, and one patient received symptomatic treatment. In 5 of 6 patients who received BT with palliative intention local control was achieved for a median time of 9.7 months (range 3-23 months).

\section{Survival data and prognostic factors}

In the univariate analysis, patients with ACC had statistically better overall survival (OS) than those diagnosed with SCC $(p=0.0001)$. Five-year OS for ACC and SCC were $77.8 \%$ and $8.4 \%$ respectively (Figure 2). Patients with performance status of 0 or 1 had better OS than 


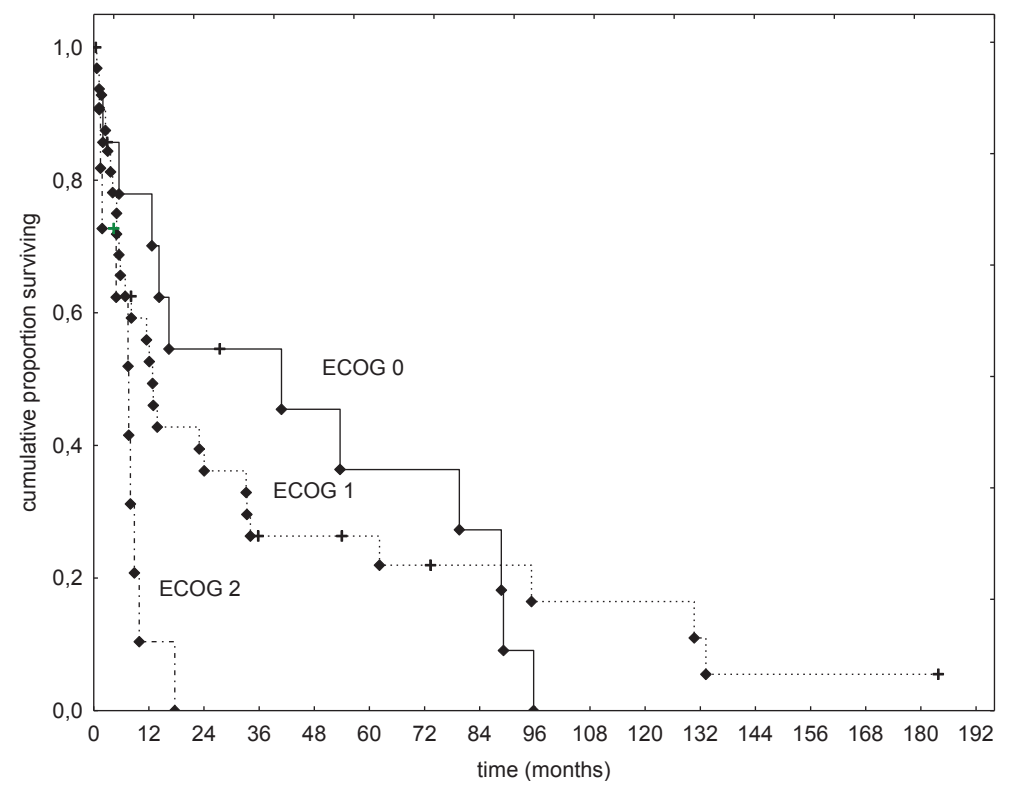

FIGURE 3. Overall survival of patients with Eastern Cooperative Oncology Group (ECOG) performance status of 0,1 , and 2 .

those with performance status of 2. Five-year OS for patients with ECOG 0 and 1 was $36 \%$ and $26 \%$, respectively, compared to $0 \%$ for patients with ECOG 2 (Figure 3 ). OS was lower in the group of smokers $(p=0.016)$. In addition, haemoptysis was a symptom associated with shorter OS ( $p=0.049)$. The presence of other symptoms did not influence the overall survival. Patients who received radiotherapy had better OS ( $p=0.026)$. Excision of the tumour (surgery or endoscopic resection) also had a positive influence on OS $(p=0.030)$. The use of systemic treatment was not proven efficient $(p=0.361)$. The varia- bles significantly influencing the OS in the Kaplan-Meier analysis were then included into a multivariate analysis which confirmed significant influence of radiotherapy $(\mathrm{p}$ $=0.013)$, performance status $(\mathrm{p}=0.033)$ and haemoptysis $(p=0.003)$ on OS. The histological type of cancer and surgical excision of the tumour did not reach the threshold of statistical significance $(p=0.068$, and $p=0.324$, respectively). The multivariate analysis did not confirm the independent significance of the smoking status $(\mathrm{p}=0.109)$.

In 11 patients (19\%, 8 men and 3 women), another cancer apart from the TC was diagnosed (Table 3). In one case, tracheal and renal tumours were diagnosed simultaneously. TC was diagnosed as a second cancer in 9 cases and in 1 as the first neoplasm. Five of them were located in the respiratory system ( 3 cancers of the larynx and 2 lung cancers). The others were: one renal cancer, one thyroid cancer, one prostate cancer, one breast cancer and one myelodysplastic syndrome.

Time between diagnoses ranged from 0 to 33.5 years with the median of 4.4 (mean 8.4). Treatment of the other cancer included: surgery in 8 cases, RT in 3 cases, and hormonal therapy in one case. Eight of these patients were diagnosed with SCC of the trachea, one patient with ACC, one patient with microcellular carcinoma, one patient with nonmicrocellular carcinoma. Six patients were current or former smokers with mean pack-years of 66.2 (range from 25 to 135).

Based on the predefined criteria we found additional 8 patients (13.8\% of the whole group) as theoretically possible surgical candidates. If nodal involvement was not considered a contraindication for surgery, the total of 15 patients met the remain-

TABLE 3. The characteristics of other cancers among patients with tracheal cancer (TC)

\begin{tabular}{ccccc}
\hline Patient No & HP of TC & $\begin{array}{c}\text { Year of diagnosis } \\
\text { of TC }\end{array}$ & HP of the other cancer & $\begin{array}{c}\text { Year of diagnosis of the other } \\
\text { cancer }\end{array}$ \\
\hline (1) & SCC & 2011 & Myelodysplastic syndrome & 2009 \\
(2) & SCC & 2006 & Larynx cancer - SCC & 1998 \\
(3) & SCC & 2005 & Clear cell renal carcinoma & 2005 \\
(4) & SCC & 2011 & Thyroid adenocarcinoma & 1978 \\
(5) & ACC & 2005 & Lung cancer - SCC & 2006 \\
(6) & Non-micro & 2013 & Clear cell renal carcinoma & 1995 \\
(7) & SCC & 2008 & Larynx cancer - SCC & 1999 \\
(8) & SCC & 1999 & Lung cancer - SCC & 1995 \\
(9) & SCC & 2003 & Larynx cancer - SCC & 2000 \\
(10) & Micro & 2010 & Prostate cancer - adenocarcinoma & 2008 \\
(11) & SCC & 2015 & Breast cancer - mucinous carcinoma & 2005 \\
\hline
\end{tabular}

$\mathrm{ACC}=$ adenoid cystic carcinoma; $\mathrm{HP}=$ histopathological type; Micro = microcellular carcinoma; No = number; Non-micro = non-microcellular carcinoma; $\mathrm{SCC}=$ squamous cell carcinoma; $\mathrm{TC}=$ tracheal cancer 
ing criteria and the percentage of potential surgery candidates rose to $25.9 \%$.

Metastases were initially diagnosed in 4 patients. During the follow-up they occurred in twelve more persons. In eleven of them, they were located in lungs, in two cases in bones, in one patient in spleen, in one case in liver and in one patient in cerebellum. In six of these patients, TC was diagnosed as SCC, in 5 patients as ACC, in two patients as microcellular carcinoma, in one patient as nonmicrocellular carcinoma, in one case as adenocarcinoma, and in one patient as papillary carcinoma.

\section{Discussion}

Epidemiological studies in Europe, USA and Japan have shown that most patients with primary malignant tracheal tumours present with an advanced local disease, have SCC tumours and are treated non-surgically.,8 Numerous authors point out that the diagnosis of TC is often delayed due to highly uncharacteristic symptoms, such as "frog in the throat" sensation reported by Howard et al. 8,10,13,18,23 In our series, one patient had been treated with surgery and RT for thyroid carcinoma until the second histopathological examination was performed and diagnosis of TC was made. Misdiagnosis of TC as thyroid tumour was also described by other authors. ${ }^{9,21}$ In their report, Licht et al. described 109 cases of TC among which 17 were diagnosed incidentally at autopsy and $21 \%$ of treated patients received asthma medication due to symptoms present at the time of diagnosis. ${ }^{13}$

ACC is usually diagnosed in younger patients than SCC and, in the current series, the median age of diagnosis of SCC and ACC also differ: 59 and 46, respectively. 4,8,10,13,21,30 ACC histology was a prognostic factor correlated with better OS in our research, which is in agreement with studies in which patients with SCC and ACC were compared. $2,7,13,15,26,27,29,31$ According to these reports, fiveyear OS for SCC ranged from $7 \%$ to $46 \%$, and for ACC from 10 to $89.4 \%$. In our group, the 5 -year OS for SCC and ACC were $8.4 \%$ and $77.8 \%$, respectively.

The other factors found to correlate with better OS were: surgery or complete resection ${ }^{4,7,15,27-32}$, radiotherapy $2,4,13,28,29,31$, the status of lymphatic nodes $7,13,14,32,33$, stage $\mathrm{e}^{7,12,34,35}$, negative airways margins $^{14,15,32}$ and the performance status. ${ }^{28,34}$

The performance status of 0 or 1 was associated with better prognosis than PS 2 in our series which is in line with previous reports. We found statisti- cally significant influence of the smoking status on OS only in the univariate analysis, however, this difference was not statistically significant in the multivariate analysis. Other studies also did not report OS to be influenced by the smoking status.

Number of patients who had undergone surgery as the first-line treatment varied among studies from $5 \%$ to $100 \%$. ${ }^{2,-8,12-17,25-27,29}$ Licht et al., in a nationwide study of TC in Denmark, and Honings et $a l$., in a nationwide study of TC in the Netherlands, reported that the number of patients that undergo surgery is $10 \%$ and $11.6 \%$, respectively. ${ }^{12,27}$ Honings and colleagues, in their multidisciplinary audit concerning undertreatment of TC, found 32\% additional surgery candidates. ${ }^{8}$ They pointed out that over $50 \%$ of TC could be treated surgically if properly diagnosed and referred to a tertiary oncology centre with multidisciplinary experience in the treatment of this disease. This is confirmed in our study. Only $25 \%$ of patients were treated invasively in our series, whereas this number could rise to about $39-51 \%$, depending on the eligibility criteria employed. Due to retrospective nature of our research, it cannot be stated that all these patients were indeed undertreated. There might have been objective factors precluding surgery which were not mentioned in the documentation available for the authors of this report. Nevertheless, this finding should be a premise to meticulous assessment of the indications and contraindications for surgery to ensure the optimal treatment of TC patients. The centralization of the care for patients with this rare airway tumour may result in selection of a greater number of patients for surgical resections and, therefore, potentially better outcomes. ${ }^{8}$

In many studies, surgery was followed by adjuvant radiotherapy (in $20-100 \%$ patients), and those who were not eligible for surgery received radiotherapy as the main treatment. The overall survival depended on whether surgery was performed, resection was complete or radiotherapy followed surgery. 2,13-15,25-27,29 Our findings indicate that surgical treatment or endoscopic resection of the tumour did not influence OS in the multivariate analysis. This somewhat surprising result can be explained by a bias introduced by low number of complete resections and low number of patients with any invasive procedures in our series. It is remarkable, however, that five-year OS for non-surgery and surgery/endoscopic resection group were $10 \%$ and $48 \%$, respectively.

Radiotherapy as a sole or main treatment method was described by a number researchers. $8,11,18,19,30-31,33-34,36$ Only four studies present the 


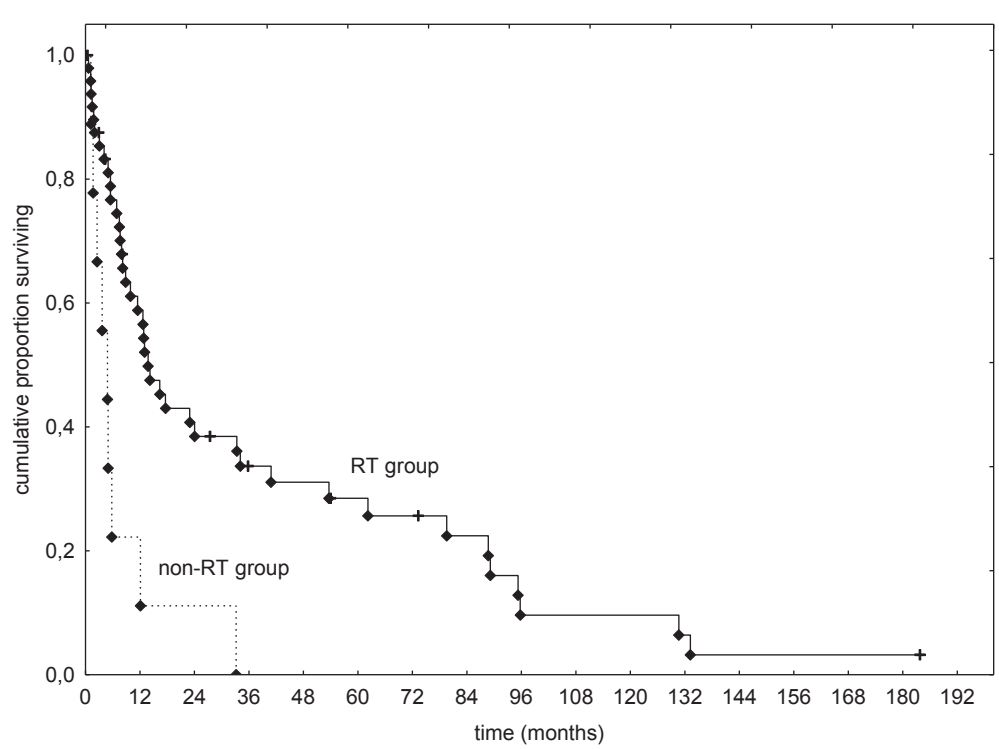

FIGURE 4. Overall survival in the irradiated (RT) and non-RT group.

outcome of at least 50 patients. $8,28,31,34$ The median total doses usually ranged from 40 to $60 \mathrm{~Gy}_{2}$. In our series, the doses delivered ranged from 14 to 82.6 $\mathrm{Gy}_{2}$ and the median total dose delivered in radical radiotherapy was $66.0 \mathrm{~Gy}_{2}$. In some reports, an additional brachytherapy boost of 6 to 30 Gy was added to escalate the dose delivered to the tumour. ${ }^{19,28,34,37,38}$ In our series, three patients had a brachytherapy boost and the BT total dose ranged from 12.5 to $16 \mathrm{~Gy}_{2}$. In the studies cited above, fiveyear OS for patients treated only with radiotherapy ranged from 8 to $30.4 \%{ }^{19,28,31,33,34}$ Mornex et al. reported that the total dose over 56 (up to 70 Gy in their series) correlated with better outcomes - 12\% for the higher TD compared to $5 \%$ for the lower. ${ }^{28}$ Hetnał et al. reported that patients with ACC histology treated with radiotherapy had better outcomes than SCC patients, and five-year OS were $80 \%$ and $9 \%$ for ACC and SCC, respectively. ${ }^{34}$ In our series, radiotherapy was a part of the treatment in 48 patients, and those patients who received RT had better overall survival in spite of large heterogeneity of the group (both radical and palliative treatments included), various fractionation schemes used, and no uniform target volume definition throughout the analysed period of time (Figure 4).

Only one of 8 patients who received brachytherapy had a haemorrhage (all others did not develop any toxicity after this treatment). Brachytherapy can be considered a part of palliative treatment like in case of bronchial infiltration in carefully selected patients. Analysis of the outcome in the current series cannot give a clear answer whether BT should be part of the treatment in all patients (for example as a boost to radical radiotherapy) because of the low number of patients in whom this treatment was employed.

There is no consensus about CTH use in TC treatment. Chemotherapy was a part of the therapy in $0 \%$ to $29 \%$ of patients in large studies. $2,12,14,25,26,28,29,31$ Cisplatin was the most commonly used drug followed by cyclophosphamide and etoposide. In our series, CTH was used in 14 patients (various regimens were employed according to diagnosed histopathologic type of TC) and 13 of them (93\%) received cisplatin-based therapy. CTH did not improve OS in our series which is similar to observation made by Manninen et al. ${ }^{31}$ However, the retrospective nature of the study and heterogeneity of the group precludes drawing unequivocal conclusions.

In the current series, TC was diagnosed as a second cancer in 10 cases, and in 1 case it was the first neoplasm. Five of the other cancers were located in the respiratory system ( 3 cancers of the larynx and 2 lung cancers). Vrabec found multiple primary carcinomas in $11.5 \%$ of 1518 patients presenting with upper aerodigestive cancers. ${ }^{39}$ Li et al. pointed out that there is a greater likelihood of developing multiple primary carcinomas in the same system than in unrelated tissues. The head and neck region and the lung are often involved with multiple primary cancers and patients with one tumour are more likely to develop a second one. ${ }^{21}$ Gaissert et al. found second cancers in almost $20 \%$ of TC patients, and most of them $(70 \%)$ were airway and lung cancers. ${ }^{15}$ Other authors found second cancers in 8 to $44 \%$ of patients (groups over 50 patients), mostly located in lungs or larynx. ${ }^{8,13,15,21-23,29}$ Histological type of the second cancer is usually the same as primary, and the SCC histology predominates.

As the number of second cancers among TC patients is relatively high, physicians engaged in the care of patients with cancer of the head and neck area should be prepared not only to treat the first malignancy, but also to be constantly vigilant for later development of subsequent primary carcinomas. ${ }^{39}$

To sum up, the diagnostic workup in TC patients should be concentrated on identification of those suitable for an invasive treatment and radiotherapy as it positively influences the outcome. Respiratory system cancer survivours can be considered a risk group for the tracheal cancer. Radiotherapy is an important part of the treatment of patients with TC, whereas the role of chemotherapy requires further investigation. 


\section{References}

1. Gomez DR, Fuller D, Chennupati S, Thomas Jr CR. Mediastinal and tracheal cancer. In: Halperin EC, Brady LW, Perez CA, Wazer DE, editors. Perez \& Brady's principles and practice of radiation oncology. Philadelphia: Lippincott Williams \&Wilkins; 2013. p. 973-95.

2. Xie L, Fan M, Sheets NC, Chen RC, Jiang GL, Marks LB. The use of radiation therapy appears to improve outcome in patients with malignant primary tracheal tumours: a SEER-based analysis. Int J Radiat Oncol Biol Phys 2012; 84: 464-70.

3. Macchiarini P. Primary tracheal tumours. Lancet Oncol 2006; 7: 83-91.

4. Webb BD, Walsh GL, Roberts DB, Sturgis EM. Primary tracheal malignant neoplasms: the University of Texas MD Anderson Cancer Center experience. J Am Coll Surg 2006; 202: 237-46.

5. Maziak DE, Todd TR, Keshavjee SH, Winton TL, Van Nostrand P, Pearson FG. Adenoid cystic carcinoma of the airway: thirty-two-year experience. $J$ Thorac Cardiovasc Surg 1996; 112: 1522-31.

6. Grillo HC. Primary tracheal neoplasms. In: Grillo HC. Surgery of the trachea and bronchi. Hamilton, London: BC Decker Inc; 2004. p. 207-48.

7. Bhattacharyya N. Contemporary staging and prognosis for primary tracheal malignancies: a population-based analysis. Otolaryngol Head Neck Surg 2004; 131: 639-42.

8. Honings J, Gaissert HA, Verhagen AF, van Dijck JA, van der Heijden HF, van Die $L$, et al. Undertreatment of tracheal carcinoma: multidisciplinary audit of epidemiologic data. Ann Surg Oncol 2009; 16: 246-53.

9. Nuwal P, Dixit R, Singhal AK. Primary adenoid cystic carcinoma of trachea presenting as midline neck swelling and mimicking thyroid tumour: a case report and review of literature. Lung India 2010; 27: 167-9.

10. Howard DJ, Haribhakti VV. Primary tumours of the trachea: analysis of clinical features and treatment results. J Laryngol Otol 1994; 108: 230-2.

11. Rosset A, Korzeniowski S. Effectiveness of radiotherapy in patients with cancer of the trachea. Nowotwory 1990; 40: 207-13.

12. Licht PB, Friis S, Pettersson G. Tracheal cancer in Denmark: a nationwide study. Eur J Cardiothorac Surg 2001; 19: 339-45.

13. Grillo HC, Mathisen DJ. Primary tracheal tumours: treatment and results. Ann Thorac Surg 1990; 49: 69-77.

14. Honings J, Gaissert HA, Weinberg AC, Mark EJ, Wright CD, Wain JC, et al. Prognostic value of pathologic characteristics and resection margins in tracheal adenoid cystic carcinoma. Eur J Cardiothorac Surg 2010; 37: 1438-44.

15. Gaissert HA, Grillo HC, Shadmehr MB, Wright CD, Gokhale M, Wain JC, et al. Long-term survival after resection of primary adenoid cystic and squamous cell carcinoma of the trachea and carina. Ann Thorac Surg 2004; 78: 188996.

16. Grillo HC. Development of tracheal surgery: a historical review. Part 1: techniques of tracheal surgery. Ann Thorac Surg 2003; 75: 610-9.

17. Grillo HC. Surgical anatomy of the trachea and techniques of resection. In Shields TW, LoCicero J III, Ponn RB, editors. General thoracic surgery. Vol 1. 5th edition. Philadelphia: Lippincott Williams \& Wilkins; 2000. p. 873-83.

18. Bonner Millar LP, Stripp D, Cooper JD, Both S, James P, Rengan R. Definitive radiotherapy for unresected adenoid cystic carcinoma of the trachea. Chest 2012; 141: 1323-6.

19. Makarewicz R, Mross M. Radiation therapy alone in the treatment of tumours of the trachea. Lung Cancer 1998; 20: 169-74.

20. Marks LB, Bentzen SM, Deasy JO, Kong FM, Bradley JD, Vogelius IS, et al. Radiation dose volume effects in the lung. Int J Radiat Oncol Biol Phys 2010; 76: 70-6.

21. Li W, Ellerbroek NA, Libshitz HI. Primary malignant tumours of the trachea. A radiologic and clinical study. Cancer 1990; 66: 894-9.

22. Jain S, Agarwal JP, Gupta T, Parikh PM, Mistry RC, Menon H, et al. Case report: Second primary small cell carcinoma of the trachea in a breast cancer survivor: a case report and literature review. Br J Radiol 2008; 81: 120-2.
23. Yasumatsu R, Fukushima J, Nakashima T, Kadota H, Segawa Y, Tamae A, et al. Surgical management of malignant tumours of the trachea: report of two cases and review of literature. Case Rep Oncol 2012; 5: 302-7.

24. Bentzen SM, Joiner MC. The linear-quadratic approach in clinical practice. In: Joiner MC, van der Kogel AJ, editors. Basic clinical radiobiology. 4th edition. London: Hodder Arnold; 2010. p. 120-34.

25. Gaissert HA, Grillo HC, Shadmehr MB, Wright CD, Gokhale M, Wain JC, et al. Uncommon primary tracheal tumours. Ann Thorac Surg 2006; 82: 268-72.

26. Urdaneta Al, Yu JB, Wilson LD. Population based cancer registry analysis of primary tracheal carcinoma. Am J Clin Oncol 2011; 34: 32-7.

27. Honings J, van Dijck JA, Verhagen AF, van der Heijden HF, Marres HA Incidence and treatment of tracheal cancer: a nationwide study in the Netherlands. Ann Surg Oncol 2007; 14: 968-76.

28. Mornex F, Coquard R, Danhier S, Maingon P, El Husseini G, Van Houtte P. Role of radiation therapy in the treatment of primary tracheal carcinoma. Int J Radiat Oncol Biol Phys 1998; 41: 299-305.

29. Regnard JF, Fourquier P, Levasseur P. Results and prognostic factors in resections of primary tracheal tumours: a multicenter retrospective study. The French Society of Cardiovascular Surgery. J Thorac Cardiovasc Surg 1996; 111: 808-13.

30. Bittner N, Koh WJ, Laramore GE, Patel S, Mulligan MS, Douglas JG. Treatment of locally advanced adenoid cystic carcinoma of the trachea with neutron radiotherapy. Int J Radiat Oncol Biol Phys 2008; 72: 410-4.

31. Manninen MP, Pukander JS, Flander MK, Laippala PJ, Huhtala HS, Karma $\mathrm{PH}$. Treatment of primary tracheal carcinoma in Finland in 1967-1985. Acto Oncol 1993; 32: 277-82.

32. Honings J, Gaissert HA, Ruangchira-Urai R, Wain JC, Wright CD, Mathisen DJ, et al. Pathologic characteristics of resected squamous cell carcinoma of the trachea: prognostic factors based on an analysis of 59 cases. Virchows Arch 2009; 455: 423-9.

33. Jeremic B, Shibamoto Y, Acimovic L, Milisavljevic S. Radiotherapy for primary squamous cell carcinoma of the trachea. Radiother Oncol 1996; 41: 135-8.

34. Hetnał M, Kielaszek-Ćmiel A, Wolanin M, Korzeniowski S, Brandys P, Małecki $\mathrm{K}$, et al. Tracheal cancer: role of radiation therapy. Rep Pract Oncol Radiother 2010; 15: 113-8.

35. Ahn Y, Chang H, Lim YS, Hah JH, Kwon TK, Sung MW, et al. Primary tracheal tumours: Review of 37 cases. J Thorac Oncol 2009; 4: 635-8.

36. Cheung AY. Radiotherapy for primary carcinoma of the trachea. Radiother Oncol 1989; 14: 279-85.

37. Harms W, Latz D, Becker H, Gagel B, Herth F, Wannenmacher M. Treatment of primary tracheal carcinoma. The role of external and endoluminal radiotherapy. Strahlenther Onkol 2000; 176: 22-7.

38. Carvalho Hde A, Figueiredo V, Pedreira WL, Aisen S. High dose-rate brachytherapy as a treatment option in primary tracheal tumours. Clinics (Sao Paulo) 2005; 60: 299-304.

39. Vrabec DP. Multiple primary malignancies of the upper aerodigestive system. Ann Otol Rhinol Laryngol 1979; 88: 846-54. 